\title{
A Glucose-Responsive Enzymatic Electrode on Carbon Nanodots for Glucose Biosensor and Glucose/Air Biofuel Cell
}

\author{
Yue Gao1,2, Guozhi Wu ${ }^{1,3}$, Feng Gao ${ }^{{ }^{*}}$ \\ ${ }^{1}$ Laboratory of Functionalized Molecular Solids, Ministry of Education, Anhui Key Laboratory of Chemo/Biosensing, \\ Laboratory of Biosensing and Bioimaging (LOBAB), College of Chemistry and Materials Science, Anhui Normal University, \\ Wuhu, China \\ ${ }^{2}$ Taishan University, Tai'an, China \\ ${ }^{3}$ College of Chemistry and Materials Engineering, Chizhou University, Chizhou, China \\ Email: ^fgao@ahnu.edu.cn
}

How to cite this paper: Gao, Y., Wu, G.Z and Gao, F. (2019) A Glucose-Responsive Enzymatic Electrode on Carbon Nanodots for Glucose Biosensor and Glucose/Air Biofuel Cell. American Journal of Analytical Chemistry, 10, 394-403.

https://doi.org/10.4236/ajac.2019.109027

Received: August 19, 2019

Accepted: September 15, 2019

Published: September 18, 2019

Copyright $\odot 2019$ by author(s) and Scientific Research Publishing Inc. This work is licensed under the Creative Commons Attribution International License (CC BY 4.0).

http://creativecommons.org/licenses/by/4.0/

\begin{abstract}
In this study, an enzymatic electrode for glucose biosensing and bioanode of glucose/air biofuel cell has been fabricated by immobilizing poly (methylene green) (polyMG) for electrocatalytic $\mathrm{NADH}$ oxidation and $\mathrm{NAD}^{+}$-dependent glucose dehydrogenase (GDH) for oxidizing glucose on carbon nanodots (CNDs). The polyMG-CNDscomposites obtained by electro-polymerization of dye MG molecules adsorbed on CNDs display excellent electrocatalytic activity toward NADH electro-oxidation at a low overpotential of ca. $-0.10 \mathrm{~V}$ (vs. $\mathrm{Ag} / \mathrm{AgCl}$ ) and the integrated enzymatic electrode shows fast response to glucose electrooxidation. Using the fabricated GDH-based enzymatic electrode, a glucose biosensor was constructed and exhibits a wide linear dynamic range from 0 to $8 \mathrm{mM}$, a low detection limit of $0.02 \mu \mathrm{M}(\mathrm{S} / \mathrm{N}=3)$, and fast response time (ca. $4 \mathrm{~s}$ ) under the optimized conditions. The developed glucose biosensor was used to detect glucose content in human blood with satisfactory results. The fabricated GDH-based enzymatic electrode was also employed as bioanode to assembly a glucose/air biofuel cell with the laccase-CNDs/GC as the biocathode. The maximum power density delivered by the assembled glucose/air biofuel cell reaches $3.1 \mu \mathrm{W} \cdot \mathrm{cm}^{-2}$ at a cell voltage of $0.22 \mathrm{~V}$ in real sample fruit juice. The present study demonstrates that potential applications of GDH-based CNDs electrode in analytical and biomedical measurements.
\end{abstract}

\section{Keywords}

Carbon Nanodots, Glucose Dehydrogenase, Laccase, Methylene Green, Biosensor, Biofuel Cell 


\section{Introduction}

It is the essential groundwork to fabricate enzymatic electrodes for constructing enzyme-based electrochemical biosensors and enzymatic biofuel cells (BFCs) [1] [2] [3] [4] [5]. Glucose-responsive enzymatic electrodes, which are responsible for electrochemical glucose biosensors or bioanodes of glucose-based BFCs with glucose as fuels, have attracted much attention [6] [7] [8] [9] [10]. Two types of commercially available enzymes, glucose oxidase (GOx) and glucose dehydrogenases (GDH), are generally employed to construct glucose-responsive enzymatic electrodes [1]-[10]. The GDH family is classified into three different types on the basis of its cofactors, nicotinamide adenine dinucleotide-dependent GDH (NAD-GDH), flavin adenine dinucleotide-dependent GDH (FAD-GDH), and pyrroloquinoline quinone-dependent GDH (PQQ-GDH) [11]-[16]. Among these enzymes, NAD-GDH is widely favored to be chosen to construct enzymatic electrode because it is insensitive to oxygen and highly specific to glucose [11]-[16]. As a result, the NAD-GDH based enzymatic electrode is allowed to improve the accuracy and selectivity of electrochemical biosensor of glucose and reduce the cross-talk of glucose/ $\mathrm{O}_{2} \mathrm{BFC}$. In order to expand the bioelectrochemical applications of NAD-GDH enzymatic electrode, extensive studies are focused on recycling the cofactor by regenerating NADH from the enzymatically produced $\mathrm{NAD}^{+}$cofactor through electrochemistry methods [11]-[20]. However, direct electrochemical oxidation of $\mathrm{NADH}$ generally requires an applied potential as high as $\mathrm{Ca} .0 .75 \mathrm{~V}$ (versus $\mathrm{Ag} / \mathrm{AgCl}$ ) although the theoretical thermodynamic potential for NADH/NAD+ couple is as low as $-0.32 \mathrm{~V}$ (versus NHE) [11] [12] [13] [14] [15]. Fast mediated oxidation of NADH using a redox enzyme and an electron mediator is required to lower the overpotential in order to reduce the interfering of glucose biosensor or improve the open-voltage of glucose $/ \mathrm{O}_{2} \mathrm{BFC}$ [11]-[20]. On the other hand, proper immobilizing materials for hosting enzymes are required to retain the bioactivity of the enzymatic electrodes [2] [3] [19] [20] [21] [22]. Up to now, various kinds of materials such as conducting polymers, metallic nanoparticles, carbon-based materials, and hybrid composites have been used as immobilizing materials to construct enzymatic electrodes [2] [3] [19] [20] [21] [22]. Among these immobilizing materials, carbon-based materials have been intrigued due to the good electric conductivity and biocompatibility [16] [18] [23] [24] [25] [26].

In this study, a NAD-GDH based enzymatic electrode was fabricated, on which NAD-GDH was immobilized on carbon nanodots (CNDs) with in-situ electrochemical polymerized methylene green (polyMG) on CNDs as electro-oxidation electrocatalyst for $\mathrm{NADH}$, to construct glucose electrochemical biosensor and the bioanode for glucose oxidation in glucose/air BFC.

\section{Materials and Methods}

Glucose Dehydrogenase (GDH, EC.1.1.1. 47, from Leuconostoc mesenteroides) and fungal laccase (EC 1.10.3.2) from Trametes Versicolor were obtained from 
Sigma Chemical Co. NADH, NAD ${ }^{+}$(disodium hydrate) was purchased from Fluka. Methylene green (MG) were supplied by No. 3 Shanghai Chemical Reagent Company. $0.10 \mathrm{M}$ phosphate buffer solution ( $\mathrm{pH}$ 7.0) was prepared by mixing the stock solutions of $0.10 \mathrm{M} \mathrm{Na}_{2} \mathrm{HPO}_{4}$ and $\mathrm{KH}_{2} \mathrm{PO}_{4}$ and adjusting the desired $\mathrm{pH}$ values with $0.1 \mathrm{M} \mathrm{NaOH}$ or $\mathrm{H}_{3} \mathrm{PO}_{4}$. Real sample fruit juice used in this study was bought from a local supermarket. All other chemicals were of analytical grade and used without further purification. $0.10 \mathrm{M}$ phosphate buffer solution ( $\mathrm{pH}$ 7.0) was used as supporting electrolyte in all electrochemical measurements unless otherwise noted. All aqueous solutions were prepared with ultrapure water $(\geq 18.25 \mathrm{M} \Omega \cdot \mathrm{cm})$ throughout the experiments.

Carbon nanodots were prepared with candle soot as starting material using the reported methods in our previous publications [18] [25] [26]. Typically, 4.0 $\mathrm{mg}$ of candle soot was suspended in $10.0 \mathrm{~mL}$ of mixed solvent $\left(\mathrm{V}_{\text {water }} / \mathrm{V}_{\text {ethanol }}=\right.$ 1:1) and sonicated for $20 \mathrm{~min}$. Then the black mixture was centrifuged with $3000 \mathrm{rpm}$ for $2 \mathrm{~min}$ to remove large-size particles. The supernatant was collected and centrifuged again for 6 min with $6000 \mathrm{rpm}$ and then a black precipitate was obtained. The obtained dry carbon nanodots were dispersed in $\mathrm{N}$, $\mathrm{N}$-dimethylformamide (DMF) under sonication of $30 \mathrm{~min}$. Finally, a black homogeneous solution with a concentration of $2.0 \mathrm{mg} \cdot \mathrm{mL}^{-1}$ was acquired for further use.

The electrochemical experiments were carried out on $\mathrm{CHI} 66^{\circ} \mathrm{C}$ potentiostat (CHI, Shanghai) with a conventional three-electrode cell. A glassy carbon (GC) electrode or modified electrode was used as the working electrode, a $\mathrm{Ag} / \mathrm{AgCl}$ as the reference electrode, and a platinum wire as the counter electrode. The electrochemical measurements were performed at room temperature and repeated minimum three times.

Glassy carbon electrodes (GC, 3-mm in diameter) surface were successively polished on polishing cloth using 0.3 and $0.05 \mu \mathrm{m}$ alumina slurry, respectively. After polishing to mirror surface, the electrodes were rinsed thoroughly with water under sonication for $3 \mathrm{~min}$ and then let them dried at room temperature. A $20 \mu \mathrm{L}$ of the CNDs dispersion was dropped on the GC electrodes. After dried under lamp, the CNDs modified electrodes (CNDs/GC electrode) were immersed into the aqueous solution of MG $(0.15 \mathrm{mM})$ for $8 \mathrm{~h}$. The electrodes were then thoroughly rinsed with distilled water to remove the adsorbed MG and polarized at $+0.85 \mathrm{~V}$ vs. $\mathrm{Ag} / \mathrm{AgCl}$ in $0.10 \mathrm{M}$ phosphate solution ( $\mathrm{pH} 7.0$ ) for $1 \mathrm{~h}$ for the electro-polymerization form polyMG/CNDs nanocomposites onto the electrodes (denoted as polyMG-CNDs/GC electrode). After that, the electrodes were taken out of the solution, thoroughly rinsed with distilled water and air-dried. The enzyme solution was prepared by dissolving $5 \mathrm{mg} \mathrm{GDH}$ in $1.0 \mathrm{~mL} 0.10 \mathrm{M}$ pH 7.0 phosphate buffer solution. $20 \mu \mathrm{L}$ aliquot of the enzyme solution dropped on the pretreated polyMG-CNDs/GC then let it dry at room temperature, and then $3 \mu \mathrm{L}$ aqueous solution of nafion $(0.5 \%$, wt $\%)$ was further coated onto the electrodes to cross-link the enzyme onto modified electrodes. 
For the assembly of glucose/air BFC, the GDH-polyMG-CNDs/GC and laccase-CNDs/GC electrode were used as bioande for glucose oxidation and biocathode for oxygen reduction, respectively. Fruit juice containing glucose was used as the fuel solution to characterize the performances of the as-prepared BFC at ambient air atmosphere. The real sample fruit juice was adjusted to $\mathrm{pH} 7.0$ with $0.1 \mathrm{M}$ PBS and the ratio for buffer solution and soft drinks was about 3:2 (v/v).

\section{Result and Discussion}

\subsection{Typical SEM and TEM Images of Carbon Nanodots}

CNDs were prepared according to previous reports [18] [25] [26]. Figure 1 shows the low magnification SEM (Figure 1(A)) and high magnification TEM (Figure 1(B)) images of the obtained CND. From the figures, we can see that the obtained products consist of a large amount of nanodots. The images reveal that the diameters of the nanodots are in the range of $40-60 \mathrm{~nm}$. The uniform nanostructures provide a significant increase in effective electrode surface for loading enzymes and accelerating electron transfer.

\subsection{Preparation and Catalytic Performances of Different Electrode}

Figure 2(A) Displays the cyclic voltammograms (CVs) recorded the in-situ electropolymerization process of monomer MG at the CNDs confined onto bare GC electrode in $0.10 \mathrm{M}$ phosphate buffer with different polymerizing time. As shown in this figure, upon being poised at $+0.85 \mathrm{~V}$ for $60 \mathrm{~min}$, the as-formed polyMG-CNDs nanocomposites show a pair of new redox wave at $-0.10 \mathrm{~V}$ with a very small peak-to-peak separation. This redox wave is originated from the reversible redox process of the as-formed polyMG at CNDs [27].

The fast and reliable detection of NADH at a low potential is of great importance in fabricating amperometric biosensors and biofuel cells based on NAD-dependent dehydrogenases [13] [14] [15] [16]. The cyclic voltammetric responses at polyMG-CNDs/GC electrodes obtained in $\mathrm{pH} 7.0$ phosphate buffer in the presence of different concentrations of $\mathrm{NADH}$ at a scan rate of $20 \mathrm{mV} \cdot \mathrm{s}^{-1}$
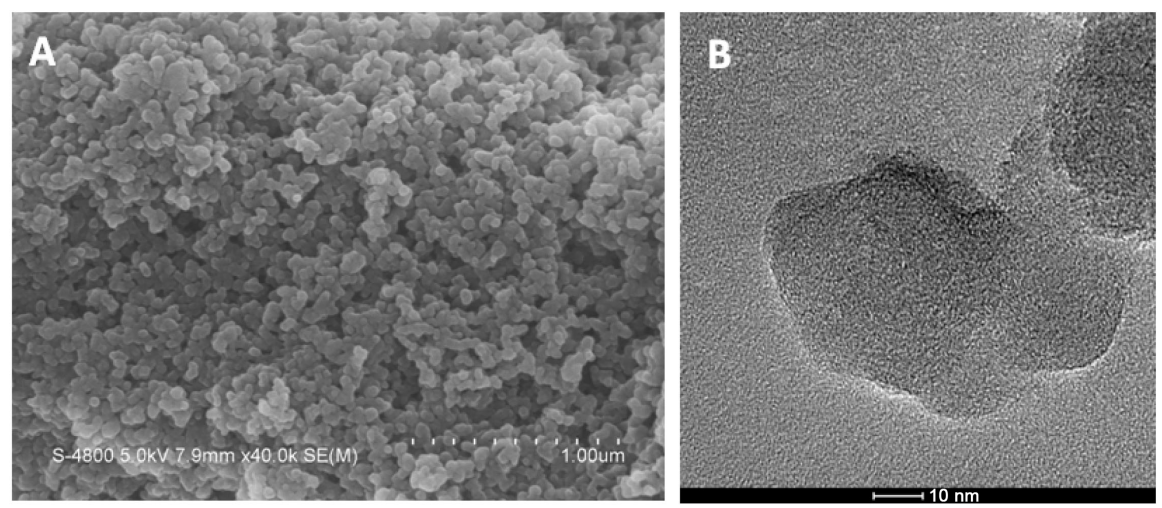

Figure 1. Low magnification SEM (A) and high magnification TEM (B) images of the obtained CNDs. 


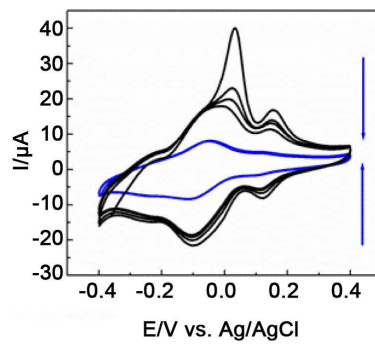

(A)

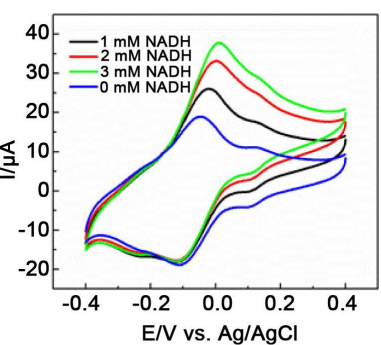

(B)

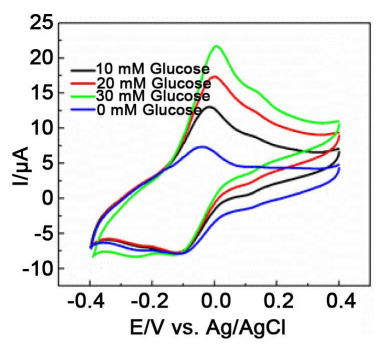

(C)

Figure 2. (A) CVs of in-situ electropolymerization of MG on CNDs/GC electrode with different time; (B) CVs obtained at the polyMG-CNDs/GC electrode in $0.10 \mathrm{M} \mathrm{pH} 7.0$ buffer solution containing $0,1.0,2.0$, and $3.0 \mathrm{mM} \mathrm{NADH}$ with a scan rate of $20 \mathrm{mV} \cdot \mathrm{s}^{-1}$; (C) CVs obtained at the GDH-polyMG-CNDs/GC electrode in $0.10 \mathrm{M} \mathrm{pH} 7.0$ buffer solution with $20 \mathrm{mM} \mathrm{NAD}^{+}$containing $0,10,20$, and $30 \mathrm{mM}$ glucose with a scan rate of 20 $\mathrm{mV} \cdot \mathrm{s}^{-1}$.

are presented in Figure 2(B). As shown in this figure, the potential for NADH oxidation at the polyMG-CNDs/GC electrode was observed at $-0.10 \mathrm{~V}$, which has a good agreement with the value reported previously [27]. These results indicate that the presence of polyMG-CNDs resulted in a substantial decrease of the overpotential to about $650 \mathrm{mV}$ for $\mathrm{NADH}$ oxidation at conventional bare GC electrode. From Figure 2(B), we also can clearly see that the peak current increases significantly with the addition of an increasing amount of NADH. These results demonstrate that the polyMG-CNDs composites show excellent electrocatalytic activity to NADH oxidation and could be further fabricated NAD-dependent dehydrogenase based enzymatic electrodes.

In order to construct a biosensor of glucose and a bioanode of glucose $/ \mathrm{O}_{2}$ $\mathrm{BFC}, \mathrm{GDH}$ was further immobilized on polyMG-CNDs composites to obtain GDH-polyMG-CNDs/GC electrode. Figure 2(C) displays the typical CVs recorded at the GDH-polyMG-CNDs/GC electrode in the presence of different concentrations of glucose with a scan rate of $20 \mathrm{mV} \cdot \mathrm{s}^{-1}$. From this figure, it is can be seen that glucose oxidation occurs at $-0.1 \mathrm{~V}$ and the catalytic oxidation current increases with the increasing concentrations of glucose.

\subsection{Real Applications of the Fabricated GDH-PolyMG-CNDs/GC Electrode}

Based on the catalytic currents dependent on the glucose concentrations, a glucose biosensor was fabricated. As shown in Figure 3(A), when GDH-polyMGCNDs/GC electrode was polarized at $-0.1 \mathrm{~V}$, successive addition of glucose to the stirring $0.10 \mathrm{M}$ PBS ( $\mathrm{pH} 7.0$ ) aqueous solution results in results in remarkable increases in the oxidation currents, and the time required to reach the steady-state current response is less than $4 \mathrm{~s}$. This amperometric response to glucose demonstrates that the GDH-polyMG-CNDs/GC modified electrode shows good sensitivity detection of glucose. Under the optimal conditions, the enzymatic electrode exhibits a wide linear dynamic range from 0 to $8 \mathrm{mM}$ with a low detection limit of $0.02 \mu \mathrm{M}(\mathrm{S} / \mathrm{N}=3)$. 


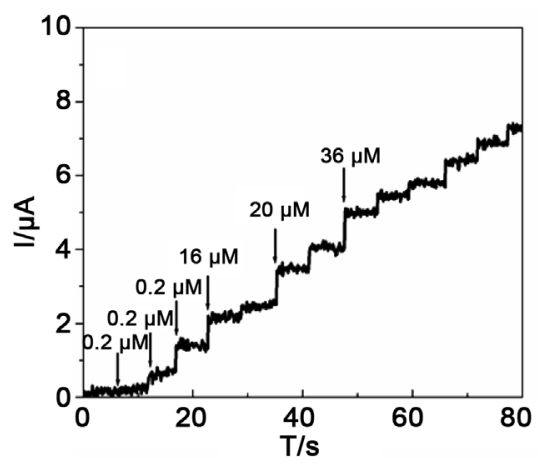

(A)

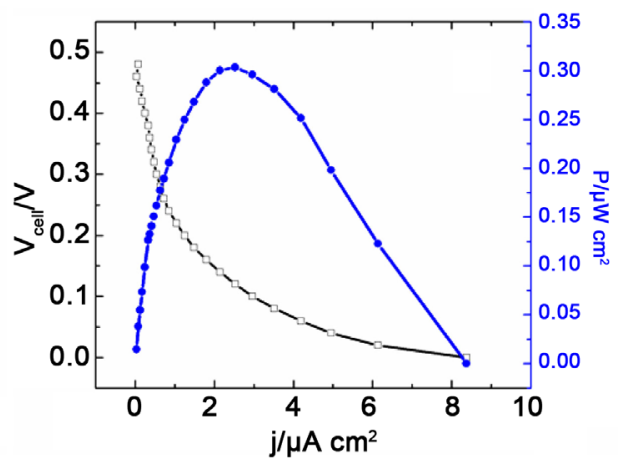

(B)

Figure 3. (A) Amperometric responses of GDH-polyMG-CNDs/GC electrode at an applied potential of $-0.1 \mathrm{~V}$ to successive addition of different concentrations of glucose in $0.1 \mathrm{M} \mathrm{pH} 7.0$ stirring buffer solution containing $20 \mathrm{mM} \mathrm{NAD}^{+}$; (B) Polarization curve (black square dots) and the dependence of power density of the assembled glucose/air BFC on current density (blue circle dots) in quiescent $0.1 \mathrm{M} \mathrm{pH} 7.0$ buffer solution containing $20 \mathrm{mM} \mathrm{NAD}^{+}$under air atmosphere.

To investigate the practical applications of the proposed GDH-based glucose biosensor in real samples, the glucose concentrations in human serum samples obtained from the Hospital attached to this University were monitored. Before measuring, the fresh serum samples were diluted using phosphate buffer solution. The determination results with the proposed biosensor and RSD values ( $\mathrm{n}$ =5) were summarized in Table 1 . From this Table, it can be seen that the determination results are good agreement with the results provided by the hospital and the RSD values are smaller than $2.97 \%$, indicating that the proposed glucose biosensor displays good accuracy and repeatability in complex samples and therefore shows potentially practical applications.

It has been attracted much attention to develop $\mathrm{NAD}^{+}$-dependent dehydrogenase based bioanodes for constructing BFCs because more than 300 dehydrogenases have been known today and therefore different substrates of dehydrogenases can be employed for biofuels [17] [18] [19]. Glucose, as a most active fuel of enzymatic BFCs, is also a common ambient fuel which exists widely in human, animals, plants, various foods. Many kinds of soft drinks, which are rich in glucose, are suitable fuels for BFCs owing to the advantages of green fuel, cheapness and availability. In this study, by using GDH-polyMG-CNDs composites as the bioelectrocatalysts for the catalytic electrooxidation of glucose at the bioanode and laccase-CNDs composites as the direct bioelectrocatalyst for oxygen reduction at the biocathode, a membrane-less glucose/air BFC was successfully constructed. The investigations on the direct electron transfer (DET) of blue-copper oxidases including bilirubin oxidase and laccase on CNDs have been studied in our previous studies [18] [26]. These studies have demonstrated that CNDs can efficiently facilitate the DET behaviours of bilirubin oxidase and laccase, and also retain their bioactivity for bio-catalyzing oxygen reduction. Based on previous studies, a laccase-CNDs enzymatic electrode was prepared as before and used as the biocathode for oxygen reduction [18] [26]. 
Table 1. Glucose determination in serum samples with the proposed glucose biosenor.

\begin{tabular}{cccc}
\hline Sample No. & Provided $(\mathrm{mM})$ & This Method $(\mathrm{mM})$ & R.S.D. $(\mathrm{n}=5)$ \\
\hline 1 & $4.37 \pm 0.02$ & $4.51 \pm 0.03$ & -1.33 \\
2 & $6.78 \pm 0.03$ & $6.70 \pm 0.01$ & 2.97 \\
3 & $5.81 \pm 0.02$ & $5.91 \pm 0.03$ & 1.47 \\
\hline
\end{tabular}

In this study, the performances of the constructed glucose/air BFC was further investigated in glucose-containing fruit juice in order to demonstrate its suitability for implantable applications. Figure 3(B) shows the polarization curve and power curve of the assembled glucose/air BFC in the quiescent fruit juice containing $20 \mathrm{mM} \mathrm{NAD}^{+}$under ambient air atmosphere in $0.1 \mathrm{M}, \mathrm{pH} 7.0$ phosphate buffer solution. The open-circuit voltage (OCV) of the BFC is ca. $0.48 \mathrm{~V}$ and the power density reaches $0.31 \mu \mathrm{W} / \mathrm{cm}^{2}$ at $0.22 \mathrm{~V}$. This result is quite comparable to that of glucose/oxygen BFC reported recently [28]. Therefore, the assembled miniature BFC can directly generate energy from soft drinks. When the cell operated continuously with an external loading resistance of $1 \mathrm{M} \Omega$ in a quiescent 0.1 $\mathrm{M}, \mathrm{pH} 7.0$ buffer solution containing $20 \mathrm{mM} \mathrm{NAD}^{+}$under ambient air, it lost ca. $9.8 \%$ of its original power in the first day and the power output remained ca. $73.4 \%$ of its original power after a week continuous work. The performance of the assembled CNDs-based glucose/air BFC is dominated by the current density of the laccase-CNDs/GC biocathode. The decreased OCV and power density may be explained by the deactivation of enzymes by some compounds within fruit juice, and low glucose and oxygen concentration in fruit juice under air atmosphere.

\section{Conclusion}

In this work, we have synthesized and characterized a carbon-based material, carbon nanodots. Using carbon nanodots as supporting matrixes,

GDH-polyMG-CNDs and laccase-CNDs were prepared and used for glucose oxidation and oxygen reduction. A glucose biosensor was fabricated with the GDH-polyMG-CNDs/GC electrode and also used for blood glucose determination in serum samples. Moreover, the GDH-polyMG-CNDs/GC electrode as a promising bioanode and DET-type laccase-CNDs /GC electrode for as biocathode four-electron reduction of oxygen were used to construct glucose/air BFC. The bioelectrocatalytic performances of the prepared enzyme electrodes using carbon nanodots as supporting matrixes were studied systematically and therefore a novel glucose/air BFC is assembled by using GDH-polyMG-CNDs/ GC electrode as bioanode and laccase-CNDs/GC as biocathode. In all, the present studies indicate that CNDs can be employed as promising immobilizing materials and electrochemical transducer in bioelectrochemistry area.

\section{Acknowledgements}

The funds from the Natural Science Foundation of China (Grant Nos. 21874001, 
21575004, 21175002), Program for New Century Excellent Talents in University (NCET-12-0599), and the Foundation for Innovation Team of Bioanalytical Chemistry of Anhui Province are acknowledged for financial supporting this work.

\section{Conflicts of Interest}

The authors declare no conflicts of interest regarding the publication of this paper.

\section{References}

[1] Kim, J., Jeerapan, I., Sempionatto, J.R., Barfidokht, A., Mishra, R.K., Campbell, A.S., Hubble, L.J. and Wang, J. (2018) Wearable Bioelectronics: Enzyme-Based Body-Worn Electronic Devices. Accounts of Chemical Research, 51, 2820-2828. https://doi.org/10.1021/acs.accounts.8b00451

[2] Wu, F., Yu, P. and Mao, L.Q. (2017) Self-Powered Electrochemical Systems as Neurochemical Sensors: Toward Self-Triggered in Vivo Analysis of Brain Chemistry. Chemical Society Reviews, 46, 2692-2704. https://doi.org/10.1039/C7CS00148G

[3] Zhao, C.E., Gai, P.P., Song, R.B., Chen, Y., Zhang, J.R. and Zhu, J.J. (2017) Nanostructured Material-Based Biofuel Cells: Recent Advances and Future Prospects. Chemical Society Reviews, 46, 1545-1564. https://doi.org/10.1039/C6CS00044D

[4] Kornienko, N., Ly, K.H., Robinson, W.E., Heidary, N., Zhang, J.Z. and Reisner, E. (2019) Advancing Techniques for Investigating the Enzyme-Electrode Interface. Accounts of Chemical Research, 52, 1439-1448.

https://doi.org/10.1021/acs.accounts.9b00087

[5] Singh, K., Mishra, A., Sharma, D. and Singh, K. (2019) Nanotechnology in Enzyme Immobilization: An Overview on Enzyme Immobilization with Nanoparticle Matrix. Current Nanoscience, 15, 234-241. https://doi.org/10.2174/1573413714666181008144144

[6] Wu, Q., Wang, L., Yu, H., Wang, J. and Chen, Z. (2011) Organization of Glucose-Responsive Systems and Their Properties. Chemical Reviews, 111, 7855-7875. https://doi.org/10.1021/cr200027j

[7] Kiyatkin, E.A. and Wakabayashi, K.T. (2015) Parsing Glucose Entry into the Brain: Novel Findings Obtained with Enzyme-Based Glucose Biosensors. ACS Chemical Neuroscience, 6, 108-116. https://doi.org/10.1021/cn5002304

[8] Nichols, S.P., Koh, A., Storm, W.L., Shin, J.H. and Schoenfisch, M.H. (2013) Biocompatible Materials for Continuous Glucose Monitoring Devices. Chemical Reviews, 113, 2528-2549. https://doi.org/10.1021/cr300387j

[9] Heller, A. and Feldman, B. (2008) Electrochemical Glucose Sensors and Their Applications in Diabetes Management. Chemical Reviews, 108, 2482-2505. https://doi.org/10.1021/cr068069y

[10] Xiao, T., Wu, F., Hao, J., Zhang, M., Yu, P. and Mao, L. (2017) In Vivo Analysis with Electrochemical Sensors and Biosensors. Analytical Chemistry, 89, 300-313. https://doi.org/10.1021/acs.analchem.6b04308

[11] Aggidis, A.G.A., Newman, J.D. and Aggidis, G.A. (2015) Investigating Pipeline and State of the Art Blood Glucose Biosensors to Formulate Next Steps. Biosensors and Bioelectronics, 74, 243-262. https://doi.org/10.1016/j.bios.2015.05.071

[12] Frias, J.P., Lim, C.G., Ellison, J.M. and Montandon, C.M. (2010) Review of Adverse 
Events Associated with False Glucose Readings Measured by GDH-PQQ-Based Glucose Test Strips in the Presence of Interfering Sugars. Diabetes Care, 33, 728-729. https://doi.org/10.2337/dc09-1822

[13] Radoi, A. and Compagnone, D. (2009) Recent Advances in NADH Electrochemical Sensing Design. Bioelectrochemistry, 76, 126-134. https://doi.org/10.1016/j.bioelechem.2009.06.008

[14] Gorton, L. (1986) Chemically Modified Electrodes for the Electrocatalytic Oxidation of Nicotinamide Coenzymes. Journal of the Chemical Society, Faraday Transactions, 82, 1245-1258. https://doi.org/10.1039/f19868201245

[15] Chenault, H.K. and Whitesides, G.M. (1987) Regeneration of Nicotinamide Cofactors for Use in Organic Synthesis. Applied Biochemistry and Biotechnology, 14, 147-197. https://doi.org/10.1007/BF02798431

[16] Moiroux, J. and Elving, P.J. (1978) Effects of Adsorption, Electrode Material, and Operational Variables on the Oxidation of Dihydronicotinamide Adenine Dinucleotide at Carbon Electrodes. Analytical Chemistry, 50, 1056-1062. https://doi.org/10.1021/ac50030a015

[17] Gao, F., Guo, X., Yin, J., Zhao, D., Li, M. and Wang, L. (2011) Electrocatalytic Activity of Carbon Spheres towards NADH Oxidation at Low Overpotential and Its Applications in Biosensors and Biofuel Cells. RSC Advances, 1, 1301-1309. https://doi.org/10.1039/c1ra00444a

[18] Wu, G., Gao, Y., Zhao, D., Ling, P. and Gao, F. (2017)Methanol/Oxygen Enzymatic Biofuel Cell Using Laccase and $\mathrm{NAD}^{+}$-Dependent Dehydrogenase Cascades as Biocatalysts on Carbon Nanodots Electrodes. ACS Applied Materials \& Interfaces, 9 , 40978-40986. https://doi.org/10.1021/acsami.7b12295

[19] Wu, G., Yao, Z., Fei, B. and Gao, F. (2017) An Enzymatic Ethanol Biosensor and Ethanol/Air Biofuel Cell Using Liquid-Crystalline Cubic Phases as Hosting Matrices to Co-Entrap Enzymes and Mediators. Journal of the Electrochemical Society, 164, G82-G86. https://doi.org/10.1149/2.0791707jes

[20] Wang, S., Yao, Z., Yang, T., Zhang, Q. and Gao, F. (2019) An Enzymatic Electrode Integrated with Alcohol Dehydrogenase and Chloranil in Liquid-Crystalline Cubic Phases on Carbon Nanotubes for Sensitive Amperometric Detection of NADH and Ethanol. Journal of the Electrochemical Society, 166, G116-G121. https://doi.org/10.1149/2.1341910jes

[21] Wen, D. and Eychmüller, A. (2016) Enzymatic Biofuel Cells on Porous Nanostructures. Small, 12, 4649-4661. https://doi.org/10.1002/smll.201600906

[22] Minteer, S.D., Atanassov, P., Luckarift, H.R., Glenn, R. and Johnson, G.R. (2012) New Materials for Biological Fuel Cells. Materials Today, 15, 166-173. https://doi.org/10.1016/S1369-7021(12)70070-6

[23] Majdecka, D. and Bilewicz, R. (2016) Nanostructuring Carbon Supports for Optimal Electrode Performance in Biofuel Cells and Hybrid Fuel Cells. Journal of Solid State Electrochemistry, 20, 949-955. https://doi.org/10.1007/s10008-015-2969-6

[24] Babadi, A.A., Bagheri, S. and Hamid, S.B.A. (2016) Progress on Implantable Biofuel Cell: Nano-Carbon Functionalization for Enzyme Immobilization Enhancement. Biosensors and Bioelectronics, 79, 850-860. https://doi.org/10.1016/j.bios.2016.01.016

[25] Sheng, M., Gao, Y., Sun, J. and Gao, F. (2014) Carbon Nanodots-Chitosan Composite Film: A Platform for Protein Immobilization, Direct Electrochemistry and Bioelectrocatalysis. Biosensors and Bioelectronics, 58, 351-358.

https://doi.org/10.1016/j.bios.2014.03.005 
[26] Zhao, M., Gao, Y., Sun, J. and Gao, F. (2015) Mediatorless Glucose Biosensor and Direct Electron Transfer Type Glucose/Air Biofuel Cell Enabled with Carbon Nanodots. Analytical Chemistry, 87, 2615-2622.

https://doi.org/10.1021/acs.analchem.5b00012

[27] Barsan, M.M., Toledo, C.T. and Brett, C.M.A. (2015) New Electrode Architectures Based on Poly(methylene green) and Functionalized Carbon Nanotubes: Characterization and Application to Detection of Acetaminophen and Pyridoxine. Journal of Electroanalytical Chemistry, 736, 8-15.

https://doi.org/10.1016/j.jelechem.2014.10.026

[28] Li, X., Zhou, H., Yu, P., Su, L., Ohsaka, T. and Mao, L. (2008) A Miniature Glucose $/ \mathrm{O}_{2}$ Biofuel Cell with Single-Walled Carbon Nanotubes-Modified Carbon Fiber Microelectrodes as the Substrate. Electrochemistry Communications, 10, 851-854. https://doi.org/10.1016/j.elecom.2008.03.019 